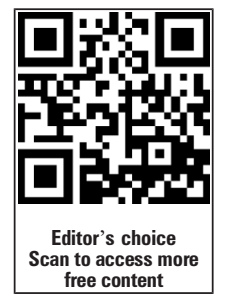

Department of Gastroenterology, Royal Alexandra \& Vale of Leven Hospitals, Paisley, Renfrewshire, UK

\section{Correspondence to}

Seth Ian Squires,

Department of Gastroenterology, Royal Alexandra \& Vale of Leven Hospitals, The Old MATCH Room Level 3 North, Floor 6 , Wards 14/27, Paisley, Renfrewshire PA2 9PN, UK; seth.squires@ggc.scot.nhs.uk

Received 16 July 2015 Revised 15 August 2015 Accepted 11 September 2015 Published Online First 9 October 2015

\section{CrossMark}

To cite: Squires SI, Boal AJ,
Naismith GD. Frontline
Gastroenterology
2016;7:216-221.

\title{
The financial impact of a nurse-led telemedicine service for inflammatory bowel disease in a large district general hospital
}

\author{
Seth Ian Squires, Allan John Boal, Graham Douglas Naismith
}

\begin{abstract}
Introduction It has become increasingly recognised that outpatient management is more cost-effective in inflammatory bowel disease (IBD). IBD Standards (Revised 2013) recommend telephone advice for patients with regard to symptoms and medication management. This report attempts to quantify the net financial impact of this service at our hospital since it was introduced in August 2013.
\end{abstract}

Method The Royal Alexandra Hospital in Paisley (National Health Service, Greater Glasgow and Clyde) is a district general hospital with a catchment population of 200000 with approximately 2500 patients with IBD. Data relating to the use of the IBD telemedicine service were prospectively recorded on a daily basis for a period of 5 months. We documented reasons for calling and the likely action taken by the patient had the telephone advice line not been available. Cost savings based on alternative outcomes were made in accordance with the Department of Health figures (Department of Health reference costs 2011-2012).

Results The mean number of calls per month was 88 (IQR 24) - (the mean number of calls which were deemed non-IBD issues was 30 calls per month (IQR 8.0)) The mean cost of staffing the IBD advice line with an IBD clinical nurse specialist was $\mathrm{f} 482.00$ per month (IQR 195.5). The mean time spent on calls per month was $28.5 \mathrm{~h}$ (IQR 11.5). Cost savings over 5 months for avoidance of general practitioner (GP) consultation was $£ 3408.00$. Savings for avoidance of a consultant appointment made over the 5month period was $£ 27$ 454.00. Savings made from patients avoiding either an accident and emergency (A\&E) or a hospital admission were $f 540.00$ and $f 11488.00$, respectively, over the 5-month period. The net saving was $£ 42890.00$.
Conclusions A nurse-led telephone advice line appears to be a cost-effective intervention. It may prevent patients from unnecessary hospital attendance. Savings can be made to both primary care and secondary care. Overall, it appears that the advice line is providing a highly valuable service not just in terms of accessible treatment decisions and guidance for patients, but cost savings when IBD clinic nurse specialist time is compared with that of GPs, consultants or hospital facilities.

\section{INTRODUCTION/BACKGROUND}

In the current healthcare economy, the implementation of telemedicine is evolving rapidly. ${ }^{1}$ It is evident that many services managing chronic illnesses such as diabetes, asthma and epilepsy have welldefined telemedicine resources. ${ }^{2}$ The reason for the development and advancement in this area seems to be twofold: first, to improve speed and efficiency in delivery of health advice and decision making, ${ }^{3}$ and second, to provide access to patients in rural areas. ${ }^{4}$

The concept of telephone triage can be traced back to Edward Chad Varah, a British Anglican priest who founded the Samaritans organisation. ${ }^{5}$ This institution offered one of the first telephone 'crisis' lines in the 1950s in order to allow people contemplating suicide to call for support and advice.

There are many examples in the evidence base of the success of telehealth strategies in terms of patient satisfaction; however, there is a gap in measuring the financial impact or cost-effectiveness of these services. ${ }^{6}$ The Department of Health reference costs ${ }^{7}$ indicate that 
non-elective hospital inpatient admissions may account for up to $26 \%$ of National Health Service (NHS) expenditure. The cost of a non-elective hospital inpatient stay, including excess bed days, is currently $£ 2160$.

It is clear from the evidence base that outpatient management is far more cost-effective in inflammatory bowel disease (IBD), ${ }^{8}$ with an emphasis placed on avoiding unnecessary admission to hospital. The National IBD Standards ${ }^{9}$ indicate that the cost of treating IBD in the UK based on 2008 prices is in the range of $£ 631-£ 762$ per patient per year. This could equate to an overall annual cost to the NHS of up to $£ 470$ million. Therefore, any initiative or strategy to reduce this cost burden would be welcomed.

\section{IBD SERVICES}

One such chronic disease that is growing in incidence is IBD. It is now considered a rising global disease, ${ }^{10}$ with many European countries estimating at least a fivefold increase in incidence over the last 30 years. Staff responsible for caring for patients with Crohn's disease and ulcerative colitis are frequently required to justify the need for resources, in order to provide the care they wish to give. The ongoing IBD National Audit ${ }^{11}$ carried out by the Royal College of Physicians has shown variability in IBD service delivery and provision.

Our IBD Service located at the Royal Alexandra Hospital in Paisley, Scotland, has attempted to show that by using an IBD telemedicine system effectively, with a clear strategy, quality IBD care can be delivered along with a significant financial impact. The savings demonstrated in our research audit can be used to justify more staff or integrated resources such as dietetics and psychology.

Cost savings aside, the audit has made us realise that using this facility effectively may well improve the long-term experience and clinical outcomes for our patients with IBD. In any future audit, we would aim to match both cost-effectiveness and patient experience; however, this study focused mainly on the financial impact.

At the centre of this process is the skill of the IBD clinical nurse specialist with the knowledge and understanding to apply an effective intervention to ensure that more costly and less effective pathways for a patient to progress to are avoided. The skill focus here is based on effective triage, appropriate diagnostic testing when required and decision making. ${ }^{12}$

The IBD Standards document ${ }^{9}$ recommends telephone advice for patients as a key resource, as patients are encouraged to report potential relapse of their disease early so that prompt assessment and treatment can be instituted. Telemedicine for IBD is key to reducing overall cost and is at the centre of the ideal care model. $^{13}$

\section{METHOD}

Our telemedicine service at the time of the audit included patients within the catchment area of the Royal Alexandra Hospital, Paisley, Scotland, only. The audit ran for 5 months in total. Patient calls were recorded on manual entry call cards prospectively.

No specific demographic characteristics were included or excluded during the audit. Patients of any age, sex, race, ethnicity, culture or creed had access to the service. However, patients who did not reside in the catchment area of the Royal Alexandra hospital with a confirmed diagnosis of IBD, either Crohn's disease or ulcerative colitis, were excluded.

Furthermore, all patients who were included required to have a continuing electronic care record at the hospital, with a named consultant gastroenterologist and registered with a general practitioner (GP) in the community. The telemedicine service was offered to both new and existing patients. The estimated population of patients with IBD confirmed by existing records and databases at the time of the audit was just under 2000.

No statistical sample size calculation was used during the time of the audit or after collating the data. On reflection, 441 calls were recorded during the 5-month audit period, which on estimate may well represent less than a quarter of the IBD population using the service. The 6-month period without an IBD telephone service, before the restart and progressive advertisement of the new telemedicine service may account for this. Further audit/study may well show further uptake and utilisation of the new service.

The reason for the patient calling was selected from a predetermined list of choices and patient identifiers, date, time of call and diagnosis were all recorded along with an outcome. A brief detail of the call was recorded, along with a duration of time required to complete the call. Time was added to the call duration if the call generated a prescription or prompted an investigation as dictated by the outcome (see figure 1).

Moreover, each patient calling in was asked whether they would have used an alternative pathway had the telemedicine service not been available. Pathway choices were Consultant Gastroenterologist Clinic (Initiative), accident and emergency (A\&E) attendance or GP appointment. All patients calling due to 'disease relapse' were assessed symptomatically for stool consistency, frequency, abdominal pain and evidence of per rectum (PR) bleeding. It should be noted that the specialist nurses did not use a specific named scoring system; however, the main elements of IBD scoring systems were used.

Objective biochemical markers such as faecal calprotectin, $\mathrm{C}$ reactive protein (CRP), erthrocyte sedimentation rate (ESR) and albumin were sought before any prescriptions were issued for additional treatments, such as steroids; however, existing treatments were 


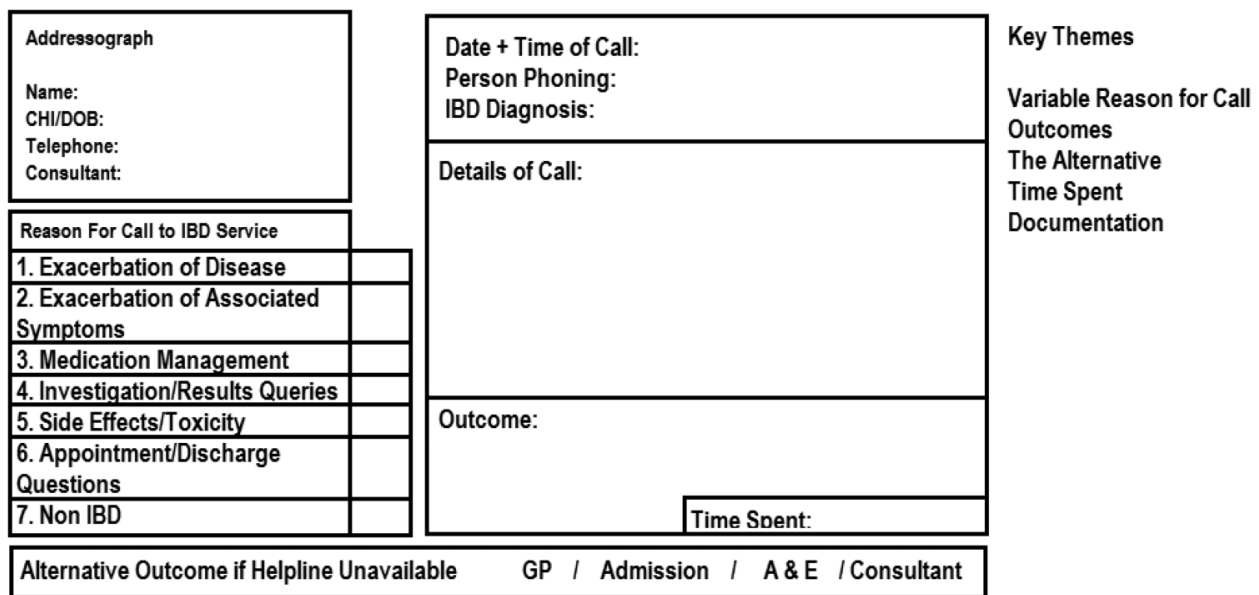

optimised. Patients who were deemed to have excessive, strong, objective and clinical evidence (raised biomarkers/faecal calprotectin) of an exacerbation of disease were marked as a potential hospital admission.

\section{RESULTS}

The Department of Health reference costs 2012$2013^{14}$ (see figure 2) were consulted along with the Royal College of Nursing (RCN) document 'Telephone advice lines for people with long term conditions'. ${ }^{15}$ Costings were then multiplied with our call cards and a net financial impact was recorded. We also collected data on the amount of calls taken and the amount of time spent on the telephone by the IBD clinical nurse specialist.

After the audit was complete, the mean number of calls per month was 88 . On average, 30 calls per month to the helpline were judged to be non-IBD issues. In terms of cost to fund the helpline, the staff cost for the IBD clinical nurse specialist was calculated as $£ 482$ per month. This was based on the mean time spent on the telephone by the IBD clinical nurse specialist of $28.5 \mathrm{~h}$ per month.

Taking into consideration the financial impact on the alternative pathways, cost savings over 5 months for avoidance of GP consultation was judged to be

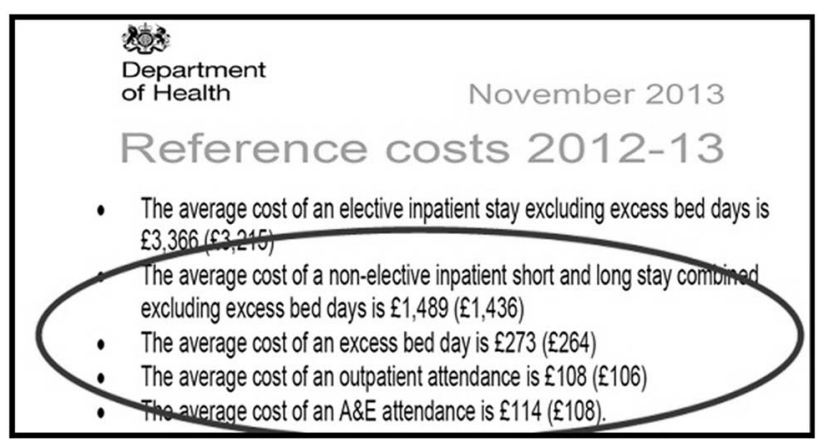

Figure 2 Department of Health reference costs. A\&E, accident and emergency.
$£ 3408.00$. Savings for avoidance of a consultant appointment over the 5-month period was $£ 27$ 454.00. Savings made from patients avoiding either an A\&E or a hospital admission were $£ 540.00$ and $£ 11488.00$, respectively, over the 5 -month period. Overall, the net saving was $£ 42890.00$ (see figure 3).

The data showed that the top reason for patients calling in was due to disease relapse, (124 calls) as one might expect. Medication management had the second highest total number of calls (98) and non-IBD-related calls came third (85 calls) over the 5 months. There was a noticeable lower cost savings in December 2013, associated with annual leave during the Christmas period, when the telemedicine service was closed.

We can confirm that the eight patients identified as avoided a hospital admission or an A\&E did not attend either accident or emergency and were not admitted for hospital treatment during the study period. The patients identified as having avoided a consultant appointment did not have a consultant clinic appointment manipulated or created after making contact with the telemedicine service. Some patients required endoscopy to assess disease extent and activity; however, these data were not collected during the audit. This would certainly be advantageous in any further audits carried out.

\section{DISCUSSION}

The implementation of telemedicine in hospitals is integral to many aspects of chronic disease service delivery which include, but not limited to, drug monitoring, referral process and education. ${ }^{16}$ The wider view adopted by the NHS is public telehealth services such as NHS 111 and NHS 24. The outcomes of these services have been well documented by $\operatorname{refs}^{17}$ and ${ }^{18}$ respectively. The triage systems that these services use mean that reductions can be achieved in inappropriate attendance at $\mathrm{A} \& \mathrm{E}$ departments. 


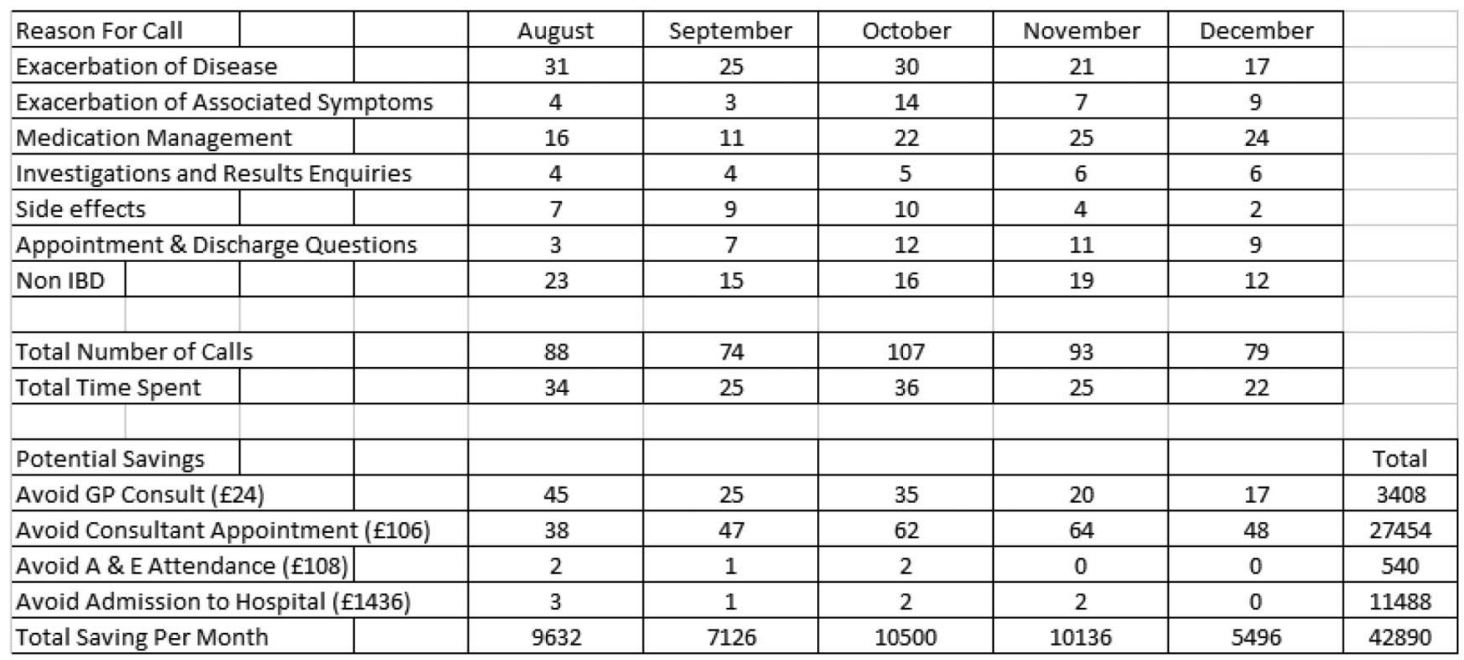

Figure 3 Extrapolated data and costings grid. A\&E, accident and emergency; GP, general practitioner; IBD, inflammatory bowel disease.

Our main aim by restarting the service was to ensure that patients would use the service for medication and symptom enquiries only, in order to effectively assess whether a patient was presenting with uncontrolled disease or exacerbation. Patients who there was no doubt had relapsed, by matching their symptom report to objective biochemical markers, were treated with escalation of therapy and in some cases glucocorticoids, ${ }^{19}$ in order to give the best possible chance of avoiding a hospital admission.

Our own experience of patients attending A\&E with gastroenteritis or constipation concerned that they may have a disease exacerbation, which prompted us to restart our telemedicine service. Recent studies have shown the value of having IBD specialists employed for this reason, ${ }^{20}$ rather than rely on either primary care or A\&E departments to make an assessment.

Cost reductions it would seem are critical in the NHS, typified by the recent 'Into the red' document published by Nuffield Trust ${ }^{21}$ indicates that the total net financial deficit from the NHS in 2013-2014 is estimated at $£ 100$ million. Nurses and doctors staffing chronic disease services are often limited with funding available to develop resources and provision for patients. Funding and 'Spend to Save Schemes' can be difficult to access in the context of an already cash strapped health service.

Within our own audit, we were acutely aware that it can be difficult to accurately predict the choice of pathway a patient may or may not take, due to the complexity of decision making and the many aspects this involves. ${ }^{22}$ However, we believe our audit shows that not only can unnecessary admission be avoided by using this type of telemedicine service effectively, that it is ultimately cost-effective. Perhaps in future, monitoring sustained remission and proven clinical outcomes with regard to the utilisation of the telemedicine service will mean the clinical benefit can be highlighted further.

Overall, the evidence base points towards telemedicine as being both effective and cost-effective and only by developing, auditing and evaluating those existing strategies can practice be improved. ${ }^{23}$ Reflecting on our own audit, using data for IBD hospital admissions matched against a robust telemedicine service may further improve the evidence base in this area. Particularly if this can be shown to coincide with swift disease control, resolution of symptoms and sustained clinical remission.

Furthermore, it is highly likely that if a patient makes a decision to call a telemedicine service looking for advice, that patient is prepared to take action to access advice of some description. Although this decision is made depending on cognitive ability, emotion and many other considerations, ${ }^{24}$ it makes sense that patients should receive advice from specialists in their disease area where available. ${ }^{25}$ If not, other facilities may refer on to specialist services regardless, creating further financial spend. ${ }^{26}$

\section{CONCLUSIONS}

Although limited by subjective analysis and determination of patient decision making, this piece of audit research clearly shows that savings can be made to both primary and secondary healthcare services. Ease of access, utility and speed of decision making are of real benefit by making use of a telemedicine support system like this.

Other limitations in our audit are a lack of focus on the improved clinical outcomes in the long term for the patients with IBD making use of the telemedicine service. However, we can confirm that the patients who contacted the service and were identified at risk during the period studied did not attend accident or emergency and were not admitted to hospital for 
treatment, which may well have happened had the service not been available. Some patients who contacted the service underwent endoscopy to assess disease extent; however, these data and the cost associated were not specifically recorded during the audit.

Further development of this service by integrating it with a patient self-management programme may well be a natural progression in future. Certainly the overarching message here is that there is a considerable value for patients who access IBD services to gain effective and accessible treatment decisions and guidance from experts in IBD management. At the point of contact, however, had the service not been available, the patients would have had used one of the more costly, non-IBD-specific pathways.

Moreover, the cost savings when specialist nurse time is compared with GPs, consultants or hospital facilities is striking. It may be that further study of these systems can provide even most robust values in terms of cost-effectiveness.

\section{Significance of this study}

\section{What is known?}

- Evidence exists that Telemedicine services are cost effective measures if managed effectively.

- Telephone services for patients are currently recommend by IBD guidelines. The specifics of how to appropriately define these telephone services does not currently exist in the literature available.

\section{What this article adds?}

- Telemedicine services for IBD, if defined as a mechanism to prevent unnecessary admission can be a cost effective system.

- It is apparent that an effective Telemedicine service can underpin the infrastructure of an IBD service.

- It is also apparent that only by appropriate resourcing and staffing could this be achieved due to the unpredictable nature of inflammatory bowel diseases.

Twitter Follow Seth Squires@SquidgeIBD

Contributors SIS was responsible for the original concept and planning of the study. SIS and AJB gathered the data for the study. SIS was responsible for drafting the manuscript. SIS, GDN and AJB revised and accepted the final version of the manuscript.

Competing interests None declared.

Provenance and peer review Not commissioned; externally peer reviewed.

\section{REFERENCES}

1 DeBlois D, Millefoglie M. Telehealth: enhancing collaboration, improving care coordination. Nurs Manage 2015;46:10-12.

2 Wootton R. Twenty years of telemedicine in chronic disease management-an evidence synthesis. J Telemed Telecare 2012;18:211-20.
3 Huang V, Reich K, Fedorak R. Distance management of inflammatory bowel disease: systematic review and meta-analysis. World J Gastroenterol 2014;20:829-42.

4 Win A. Telehealth can bridge the gap for rural, disabled, and elderly patients. J Am Med Dir Assoc 2015;16:268-9.

5 Constant A, Platt B. Someone to talk to: The story of Chad Varah and the Samaritans (Faith in Action). Religious and Moral Education Press, 2002.

6 Ellis A. An Evaluation of Patient Satisfaction with Telephone Follow-up in an Urgent Care. Diss. Otterbein University, 2015.

7 Department of Health. Reference Costs 2012-2013. https:// www.gov.uk/government/uploads/system/uploads/attachment data/file/261154/nhs_reference_costs_2012-13_acc.pdf (accessed Jul 2015).

8 Park KT, Bass D. Inflammatory bowel disease-attributable costs and cost-effective strategies in the United States: a review. Inflamm Bowel Dis 2011;17:1603-9.

9 The IBD Standards Group. IBD Standards Updated, 2013. http://www.ibdstandards.org.uk/uploaded_files/ibdstandards.pdf (accessed Jul 2015).

10 Molodecky N, Soon S, Rabi D, et al. Increasing incidence and prevalence of the inflammatory bowel diseases with time, based on systematic review. Gastroenterology 2012;142: 46-54.

11 Royal College of Physicians. IBD National Audit. https://www. rcplondon.ac.uk/projects/inflammatory-bowel-disease-audit (accessed Jul 2015).

12 Greveson K, Woodward S. Exploring the role of the inflammatory bowel disease nurse specialist. Br J Nurs 2013;22:952-8.

13 Sack C, Phan V, Grafton R, et al. A chronic care model significantly decreases costs and healthcare utilisation in patients with inflammatory bowel disease. J Crohns Colitis 2012;6:302-10.

14 Department of Health. Reference Costs 2012-2013. https:// www.gov.uk/government/uploads/system/uploads/attachment_ data/file/261154/nhs_reference_costs_2012-13_acc.pdf (accessed Jul 2015).

15 Royal College of Nursing. Telephone advice lines for people with long term conditions: guidance for nursing practitioners, 2006. https://www.rcn.org.uk/_data/assets/pdf_file/0008/ 78695/003033.pdf (accessed Jul 2015).

16 American Telemedicine Association. What is telemedicine? 2014. http://www.americantelemed.org/about-telemedicine/ what-is-telemedicine (accessed Jul 2015).

17 Turner J. National Health Service NHS 111 Final Report. 2012. http://www.sheffield.ac.uk/polopoly.../NHS_111_final_ report_August_2012.pdf (accessed Jul 2015).

18 Auditor General for Scotland. NHS 24 Report to the Board. 2013. http://www.audit-scotland.gov.uk/docs/health/2013/ fa_1213_nhs_24.pdf scott-moncrieff.com (accessed Jul 2015).

19 De Cassan C, Fiorino G, Danese S. Second-generation corticosteroids for the treatment of Crohn's disease and ulcerative colitis: more effective and less side effects? Dig Dis 2012;30:368-75.

20 Bennett A, Munkholm P, Andrews J. Tools for primary care management of inflammatory bowel disease: Do they exist? World J Gastroenterol 2015;21:4457-65.

21 Lafond S, Arora S, Charlesworth A, et al. Research reportThe NHS Into the Red, 2014. http://www.nuffieldtrust.org.uk/ sites/files/nuffield/publication/into-the-red-report.pdf (accessed Jul 2015).

22 Tulsky J. Decision aids in serious illness: moving what works into practice. JAMA Intern Med 2015;175:1221-2. 
23 Elbert NJ, Van Os-Medendorp H, Renselaar W, et al. Effectiveness and cost-effectiveness of ehealth interventions in somatic diseases: a systematic review of systematic reviews and meta-analyses. J Med Internet Res 2014;16:110.

24 Boardman CR, Sonnenberg A. Gut instincts: my perspective. Clin Transl Gastroenterol 2014;5:63.
25 Niv Y, Dickman R, Levi Z, et al. Establishing an integrated gastroenterology service between a medical center and the community. World J Gastroenterol 2015;21:2152-8.

26 Ekeland A, Bowes A, Flottorp S. Methodologies for assessing telemedicine: a systematic review of reviews. Int J Med Inform 2012;81:1-11. 